\title{
Vivre en français en France - Des japonais qui se cherchent
}

Entretiens avec Sa et Shu, étudiants japonais

\section{Kuri Komatsu}

\section{OpenEdition}

\section{Journals}

Édition électronique

URL : http://journals.openedition.org/esp/435

DOI : $10.4000 /$ esp. 435

ISSN : 2532-0319

\section{Éditeur}

Centre d'Information sur l'Éducation Bilingue et Plurilingue

Édition imprimée

Date de publication : 1 décembre 2014

Pagination : 55-66

ISSN : 1127-266X

\section{Référence électronique}

Kuri Komatsu, «Vivre en français en France - Des japonais qui se cherchent », Éducation et sociétés plurilingues [En ligne], 37 | 2014, mis en ligne le 01 octobre 2015, consulté le 20 avril 2019. URL : http:// journals.openedition.org/esp/435; DOI : 10.4000/esp.435 


\section{VIVRE EN FRANÇAIS EN FRANCE - DES JAPONAIS QUI SE CHERCHENT}

\section{Kuri Komatsu}

Molti giovani giapponesi vanno a "studiare" in paesi stranieri. Tuttavia l'apprendimento della lingua del paese che li ospita e il prosieguo degli studi all'estero non costituiscono sempre $i$ loro unici obiettivi. Alcuni giapponesi vanno in un paese straniero al fine di effettuare una "ricerca di sé". Si parla di "viaggio alla ricerca di se" (jibun sagashi no tabi), un'espressione di moda presso i giovani giapponesi di oggi. Quest'articolo si basa su un'intervista a due studenti: una di essi ha preso un anno sabbatico per venire in Francia con un visto vacanzalavoro; l'altro è venuto a studiare il francese a Strasburgo. Vorremmo capire perché siano venuti a vivere in Francia in questo periodo.

Many young Fapanese people go to "study" abroad. However, learning the language of the foreign country or studying there are not always their only objectives. Some Fapanese leave in order to "search for oneself". As the saying goes, they "travel in search of self" (jibun sagashi no tabi), the expression is fashionable among the young people today. This article is based on an interview with two students; one took a year's leave to come to France on a Vacation-Work visa, the other came to learn French in Strasburg. The author tries to understand why they chose to come to France at this time.

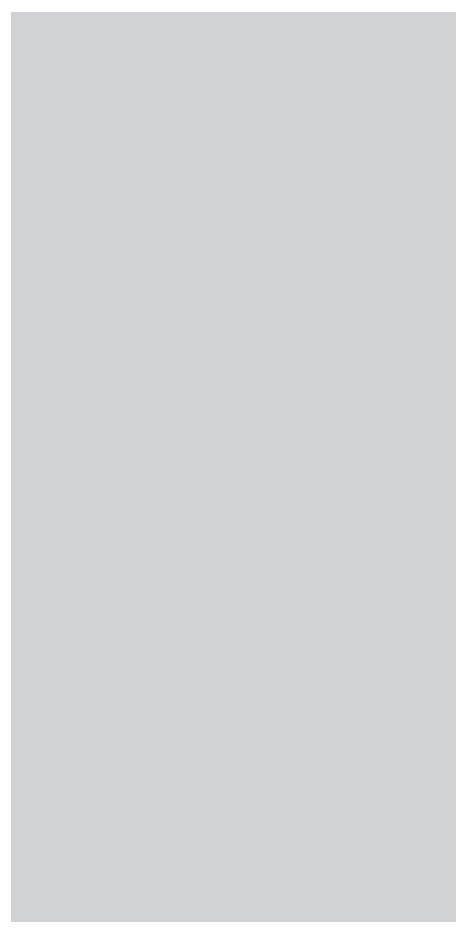

自分探しの旅 ‘jibun sagashi no tabi’ Le voyage de recherche de soi. C'est une expression qui est dans l'air du temps parmi les jeunes de la société japonaise d'aujourd'hui. L’idée existe depuis longtemps au Japon; selon Nakama (2008), la recherche de soi (自分探 し jibun sagashi) désigne le processus par lequel les jeunes gens essaient de comprendre qui ils sont réellement. Pour cela, ils ont tendance à faire quelque chose d'inhabituel, qu'il s'agisse d'un voyage à l'étranger, d'études à l'étranger, d'un travail nouveau, ou autre chose. Furukawa (2005) estime qu'il s'agit d'un concept important pour décrire la société japonaise moderne, renvoyant à la recherché de sa propre identité. La popularité actuelle du voyage de recherche de soi est relativement récente, bénéficiant de l'exemple de célébrités comme le joueur japonais de football Hidetoshi Nakata (1).

Nakama (2008) estime que les jeunes japonais effectuent une recherche de soi à cause de leurs sentiments d'inconfort par rapport à la société, et de leur désir de développement personnel. 
Vivre en français en France Des japonais qui se cherchent

K. Komatsu
Ono (2011) écrit que l'un des moyens de formation d'une identité individuelle est le voyage, où des jeunes gens entrent en contact avec des cultures variées pendant de longues périodes de temps. Or, la société japonaise actuelle attend de ses jeunes qu'ils fassent preuve d'une identité individuelle marquée, motivant ces derniers à effectuer un voyage de recherche de soi. Cette attente est liée à la transformation de la société japonaise en une «nouvelle société libérale», et à la perte des emplois individuels stables («à vie»).

Mizuma (2006) a expliqué que les individus tendent à trouver leur individualité (jibun rashisa: se trouver)* et comment ils peuvent 'être eux-mêmes' dans leur vie quotidienne. Cela va à l'encontre de la société japonaise où on évite de montrer son individualité, et où le groupe est favorisé. Cependant, selon Ohta et Ishino (2011), cela ne se limite pas à la recherche de soi pour les étudiants en université, mais comprend aussi la recherche de ce qu'ils veulent faire dans le futur après leurs études, et la découverte de leur identité pouvant déboucher sur un travail futur. On peut aussi dire qu'en accomplissant quelque chose, les étudiants cherchent à se comprendre avant de commencer à travailler.

L'expression «le voyage de recherche de soi» a été remplacée par «les études dans un pays étranger pour la recherche de soi» parce que la deuxième expression sonne mieux: elle suggère qu'on fait quelque chose d'autre que de voyager. En séjournant à l'étranger, les étudiants japonais peuvent devenir plus indépendants, et essayer de trouver leur vraie nature avant de commencer à travailler.

Dans un article publié par l'Ambassade des États-Unis au Japon, un étudiant japonais a écrit un article parlant de son expérience d'études aux États-Unis sous le titre Le voyage de recherche de soi (jibun sagashi no tabi) (2), écrivant:

«Lorsque je suis allé aux États-Unis, mes parents m'ont dit 'Trouve ce que tu veux faire'. En étudiant dans une université américaine, je me demande ce que j'ai trouvé, et je me suis cherché (jibun sagashi) du début à la fin».

Même dans les programmes d'études à l'étranger dans les universités japonaises, on parle de «projet de voyage de recherche de soi 120» (LMP120, Looking for Myself Project 120) 'jibun sagashi no tabi no project 120 ' à l'université féminine de Fukuoka.

Il ne s'agit pas d'une simple expérience de séjour à l'étranger, c'est une expérience conçue pour découvrir ce qu'ils doivent apprendre à l'université via le stage, et réfléchir à leur futur et à leur relation avec la société internationale. C'est un projet qui conduit plus tard à une expérience d'étude à l'étranger de longue durée (3). 
Vivre en français en France Des japonais qui se cherchent

K. KomatsU
INTERVIEW AVEG UNE ÉTUDIANTE JAPONAISE, SA
Yasuhiko Koyama (4) a écrit au sujet de son expérience d'études dans une université américaine qu'il avait étudié dans une université américaine pendant deux ans pour se chercher, et pour mûrir. Cependant, des critiques ont dit aussi que «faire des études dans un pays étranger pour la recherche de soi» n'est rien de plus qu'un moyen de fuir la réalité: «Si les jeunes japonais ne sont pas sûrs d'eux, il doit être plus difficile de rester à l'étranger, où ils seront d'avantage interrogés au sujet d'eux-mêmes en tant que personne, en tant que japonais... etc. S'ils ne sont pas sûrs d'euxmêmes au Japon, alors c'est une illusion que de penser qu'ils peuvent se trouver à l'étranger où ils ont encore plus de difficultés dans leurs vies quotidiennes».

Pour les étudiants japonais, l'apprentissage du français est l'une des raisons de leur venue en France, mais n'est pas leur but principal. Christophe Dreyer (5), directeur de l'Alliance Française de Nagoya, explique que contrairement aux étudiants chinois qui étudient le français en vue d'études futures à l'université en France, les Japonais étudient le français comme un loisir, sont intéressés par la culture et la gastronomie françaises, mais ne désirent pas améliorer leur niveau de français au-delà du niveau B2. Donc les étudiants japonais viennent en France, mais il se peut que l'apprentissage du français ne soit qu'un à-côté de leur voyage, et qu'ils viennent en France pour d'autres raisons, par exemple pour rechercher leur identité. De plus, pour des étudiants japonais, apprendre une langue n'est pas l'objectif principal de leur séjour dans un pays étranger, et il se peut qu'ils n'étudient pas assidument. Même s'il s'agit d'une bonne expérience, ce n'est pas toujours une expérience positive les aidant à trouver un travail ou à s'engager dans une carrière.

J'ai eu l'occasion de rencontrer SA, une étudiante japonaise à l'université, venue en France avec un visa vacances-travail lui permettant de rester et de travailler en France pendant un an.

SA est une étudiante en $4^{\text {ème }}$ année de philosophie à l'Université de Hôsei à Tokyo. Elle a pris une année de pause (kyuka: sabbatique). Son université organise un séminaire avec l'Université de Strasbourg, et elle était déjà venue à Strasbourg en 2012. Il s'agissait alors de son premier voyage à l'étranger. Cette fois-ci, elle est arrivée en France en mai 2013, et y est restée pendant un an. Elle est allée à une école de langue française à Nice pendant les six premiers mois pour pratiquer le français, et ensuite, elle est venue à Strasbourg où elle voulait travailler pour gagner sa vie pendant les six derniers mois. 
Vivre en français en France Des japonais qui se cherchent

K. KomatsU

PREMIER ENTRETIEN AVEG SA
- Pourquoi êtes-vous venue en France? Pourriez-vous me dire le but de votre venue en France?

SA: Simplement, j'aime les mots, et j'aime réfléchir aux discordances entre les mots et les émotions, je pensais qu'on pouvait aussi trouver de telles discordances entre la langue maternelle et une langue étrangère, et je voulais faire l'expérience de vivre dans une langue étrangère. C'est mon excuse (rire) Et, la raison principale... Je n'ai jamais eu de véritable sentiment d'échec (zasetsu kan) et je suis venue ici pour être bousculée (momareru) (6). C'est une raison un peu frivole, mais si je rejoins la société immédiatement, ce pourrait être encore plus frivole. Et lorsque je commencerai à travailler, je ne sais pas quand j'aurai à nouveau une telle occasion [de venir en France], donc j'ai pensé que c'était maintenant ou jamais.

- Pour vous, que signifie votre séjour d'un an en France? S'agit-il d'une année de séjour à l'étranger? Si vous dites que la raison pour laquelle vous vouliez vivre à l'étranger était d'être bousculée...

SA: Ce sont peut-être des vacances. Je peux aussi dire que c'est pour éviter de chercher un travail. Ce ne sont pas de bonnes raisons. Mon visa est appelé visa vacances-travail... Cependant, j’ai pu goûter d'un échec (zasetsu) en allant à l'école de langue. La barrière de la langue. Je m'y attendais, et c'était bien (rire) c'est un peu étrange à dire.

- Mais vous parlez d'un échec (zasetsu)... Vous attendiez-vous à faire l'expérience d'une déception? Vous avez aussi dit que vous n'aviez jamais fait l'expérience d'un échec (zasetsu).

SA: Oui. Mon français n'est pas au niveau 3 du Diplôme d'Aptitude Pratique au français, et je pensais que j'avais un problème de langue, et je voulais faire tomber le mur des mots (kotoba no kabe ni buchiataru: faire tomber la barrière de la langue). Je ne suis pas de nature pessimiste au point de m'inquiéter d'avoir une déception (rire). J'ai tenté uniquement l'examen d'entrée à mon premier choix de lycée, et heureusement j'ai réussi cet examen. Je n'ai que des problèmes d'amitié et n'ai jamais eu de problème sérieux. Comment dire, je suis très incertaine... et je pense que je voulais avoir une expérience difficile (tsurai). Donc quand je rencontre des Japonais qui s'amusent, j'ai une impression étrange. Je ne suis pas si sérieuse, mais j'ai l'impression de 'me' voir dans la glace (rire).

- C'est-à-dire... rester dans un pays étranger pour faire une expérience difficile? Passer un moment difficile? Ou bien vous voulez essayer de faire quelque chose, ou faire de votre mieux... aller en 
Vivre en français en France Des japonais qui se cherchent

K. Komatsu
France pour essayer de faire des efforts (ganbaru) (7)...

SA: Je peux dire que ce sont les deux! Non... pour être honnête, au départ il s'agissait de passer un moment difficile. Mais ensuite j'ai pensé que c'était la première fois que je me concentrais pour faire quelque chose. Le but (de mon séjour en France) est pour l'expérience, et par conséquent je me concentre pour ce but.

- Donc, quel est votre but en venant en France?

SA: L'expérience... Je voulais faire quelque chose que je n'avais jamais fait. Et je voulais être dans une situation d'où je ne pouvais pas m'échapper. Je m'enfuis toujours avant d'avoir une déception, ou je cherche toujours à éviter les situations difficiles, donc je voulais me heurter à la réalité (genjitsu ni butsukaru: confronter à la réalité) avant de rejoindre la société réelle. Cela reste plus facile que la réalité (rire). Je peux dire que mon but était d'essayer mes bras (ude dameshi: de me mettre à l'épreuve).

- Vous vouliez faire quelque chose que vous n'aviez jamais fait. Que décririez-vous comme ce que vous n'avez pas fait? Et quelle est la situation à laquelle vous ne pouvez pas échapper? S'agit-il de la vie en France? Ou de la vie dans une langue étrangère?

SA: Ce que je n'ai jamais fait... C'était pour vivre loin de mes parents et du Japon, parce que mon premier voyage à l'étranger était en février 2012, et pour vivre dans une langue étrangère. La situation d'où je ne peux pas m'échapper est une situation où je ne peux pas abandonner (rire). Même si j'ai une vie difficile et que je m'ennuie, je ne peux pas revenir au Japon facilement. Bien sûr, je peux réserver un billet d'avion pour revenir à la maison mais ce n'est pas simple et je dis «je ne peux pas m'enfuir».

- Mais pourquoi la France? Pourquoi avez-vous décidé d'aller en France? Avez-vous une raison pour venir en France? J'ai l'impression que vous vouliez vivre dans un pays étranger. Y avait-il une raison pour que ce soit la France?

SA: C'est parce que la France est le premier pays étranger où je suis allée. Je suis tombée amoureuse de Strasbourg. Et j’aime la langue française. C'est là la raison pour laquelle je fuyais. Jusqu'à ma deuxième année d'université, je n'étais pas intéressée par les pays étrangers, et lorsque j'ai commencé à réfléchir à faire des études à l'étranger, mon niveau d'anglais n'était pas assez bon. J'avais étudié l'allemand comme seconde langue mais c'était difficile, et je me suis intéressée au français que j'avais étudié, donc j'ai pensé à essayer d'étudier en français.

- Donc, comment est la vie en fait en France?

SA: Eh bien... Ce n'est pas pareil à Nice et à Strasbourg, mais c'est difficile. Non, ce n'est pas difficile... mais c'est satisfaisant, 
Vivre en français en France Des japonais qui se cherchent

K. Komatsu j'apprécie! C'était difficile de s'habituer à la langue à Nice. Bien que j'aie étudié, j'avais l'impression que tout le monde parlait très vite. Mais j'ai étudié pour apprendre plus de vocabulaire, et, au fur et à mesure que je m'habituais à la langue, je comprenais de plus en plus, et parler devenait de plus en plus amusant. Je suis ensuite arrivée sans activité à Strasbourg... Il faut que je trouve quelque chose à faire, et je suis occupée pour remplir tous les documents, mais je suis excitée parce que la ville est belle.

- Vous avez dit que vous aviez eu une expérience de grand échec (ôkina zasetsu)?

SA: Quand j'étais à l'école de langue, il y a eu un moment où j'étais perdue alors que la conversation continuait et tout le monde riait. Je ne comprenais pas du tout pourquoi tout le monde riait et ce dont ils parlaient, donc je suis revenue chez moi les larmes aux yeux (rire). Si ç'avait été le début, j’aurais pu penser «c'est comme ça», mais c'était déjà mon quatrième mois...

- Donc la principale raison pour cet échec était la langue?

SA: J'étais déçue parce que je ne comprenais pas bien même après avoir passé quatre mois. Je ne veux plus être déçue. Je vais faire des efforts.

- Le mur des mots?

SA: C'était précisément le mur des mots. Il est encore haut...!

- Que voudriez-vous faire à l'avenir en français? Ou plutôt, est-ce que vous voudriez faire quelque chose en français? Ou bien, comme le but de ce séjour était de vivre dans un pays étranger, vous n'avez pas d'objectif futur?

SA: Je ne pense pas à travailler en français pour le moment. Je pense que la langue française est simplement un de mes passetemps (shumi: passe-temps).

- Après votre séjour en France, qu'aimeriez-vous faire? Avez-vous un rêve ou un objectif après votre séjour en France?

SA: Après mon séjour en France, je serai une étudiante en $4^{\text {ème }}$ année d'université, donc je finirai mes études et trouverai un travail. Ce n'est pas ce que je veux faire, mais je devrais dire que c'est ce que je pense que je «devrais» faire. Terminer mes études, puis trouver un travail, ou même, je termine mes études, je ne peux pas trouver de travail et je dois en chercher un... Je n'ai jamais réfléchi à des alternatives. Mais quand je pense au chômage, je me dis que je veux terminer mes études à l'université et trouver un travail. Je pense que je veux faire quelque chose de toute façon. Mon rêve ou objectif pour le futur est... difficile (rire). Je voudrais avoir un travail lié à la maison... et je voudrais avoir une famille heureuse. 
Vivre en français en France Des japonais qui se cherchent

K. KomatsU

\section{DEUXIÈME \\ ENTRETIEN AVEG SA}

- Vous avez dit «Je n'ai jamais eu de véritable sentiment d'échec, et je suis venue ici pour être bousculée.» Pourquoi fallait-il que ce soit à l'étranger? Ou en France? Par exemple, pourquoi pas au Japon? Si on parle d'"être bousculée", on pense à d'autres pays difficiles.

SA: Bien sûr, il y a d'autres pays difficiles! Je n'ai pas beaucoup réfléchi (rire). Je peux communiquer en japonais au Japon et je ne peux pas y être seule, donc j'ai pensé que cela pourrait être plus difficile dans une langue étrangère, et j'ai choisi la France parce que c'est un pays qui m'intéressait. Oui, je suis venue ici parce que la France m’intéressait. J'aimerais penser... Je ne peux pas comprendre la langue, je ne peux pas m'exprimer comme je le désire, c'est mentalement difficile, donc je peux envisager de faire des efforts. Je suis très optimiste.

- Vous voulez simplement parler de sentir des difficultés à se faire comprendre par la langue? J'imagine que beaucoup de gens ont un véritable traumatisme, à cause du tremblement de terre et du tsunami, mais peut-être pas à Tokyo...

SA: Je ne parlais que des difficultés pour se faire comprendre par la langue. Ce sont des difficultés qu'on peut compenser par l'effort.

- Je vois, il ne s'agit donc pas de difficultés insurmontables, mais de difficultés où on peut faire des efforts pour les surmonter. Estce que c'est juste?

SA: C'est juste! Je ne veux pas goûter (ajïwau: faire l'expérience) à des difficultés contre lesquelles je ne peux rien faire (rire).

- Donc, pour faire l'expérience d'un certain niveau de difficulté?

SA: Oui.

- Donc, pourquoi la langue? Il y a de nombreux types de difficultés... Les difficultés liées à la compréhension ne sont pas les seules, vous avez dit que la France était un pays qui vous intéressait... J'ai l'impression que vous n'êtes pas venue ici seulement pour avoir une expérience de difficultés.

SA: Bien sûr! Avoir une expérience difficile n'est pas l'objet principal, donc je n'ai pas beaucoup de choses à répondre à ce sujet. $\mathrm{Si}$ je ne peux pas parler la langue, je ne peux pas vivre, donc je voulais me placer dans cette situation.

- Bien, la dernière fois vous avez parlé de faire l'expérience d'une «véritable déception». J'imagine que c'est inhabituel pour des visiteurs étrangers de chercher à avoir une expérience difficile. Pourquoi cherchez-vous cela?

SA: Donc, il se peut que cela soit une exagération de parler d'une véritable déception. Comment dire... Mais ce serait mauvais de 
Vivre en français en France Des japonais qui se cherchent

K. Komatsu
ENTRETIENAVEG UN ÉTUDIANTJAPONAIS, SHU m'intégrer à la société tout de suite. Ce n'est pas bien d'être vide (sukkarakan: d'être sans but, sans motivation), donc je pense que j'aurai une expérience qui me demande de faire des efforts, et ainsi faire quelque chose dont je pourrai dire que j'ai fait des efforts pour cela... et ensuite je finirai mes études et trouverai un travail...

\section{- Etre vide? Pourquoi êtes-vous vide?}

SA: Comment je peux dire, je suis vide... (rire). J'obtiens un diplôme, et ce n'est pas vide, mais ce n'est pas satisfaisant. J'imagine que ça ne suffit pas... Je pense que je veux ce que je n'ai pas (rire).

- Mais j'imagine que c'est assez courant au Japon? Comme pour la recherche de soi, par exemple... rechercher son identité... pourquoi cela?

SA: Je ne sais pas, pour «la recherche de soi», donc je ne peux pas dire grand-chose. Je voulais faire quelque chose que je n'avais jamais fait avant de m'intégrer à la société. C'est tout.

- Après un an de séjour en France, eh bien, vous en êtes déjà à la moitié... Pourrez-vous accomplir votre objectif?

SA: Oui! Je pleure de dépit... au sujet du travail, je ne suis pas sûre mais je vais avoir un entretien... Si je peux travailler ici je pourrai avoir une autre bonne expérience. Si je ne peux pas trouver un travail... C'est comme ça... eh bien, je ne sais pas.

- Si vous restez en France pendant un an, y a-t-il quelque chose à changer? Vous dites que vous ne seriez pas vide.

SA: Comment est-ce que je serai? Je n'ai pas de grandes attentes. Mais de bons souvenirs? Si je peux me dire «Ah, j’ai fait des efforts, j'ai fait de mon mieux», alors je ne serai pas vide (rire).

- Donc cela veut dire que vous êtes venue ici non pour expérimenter une véritable déception, mais pour faire de votre mieux, pour accomplir quelque chose?

SA: C'est vrai, on peut dire ça comme ça. Comme j’ai dit, une véritable déception est quelque chose qui peut être changé avec des efforts en France. Oui, je suis venue ici pour faire de mon mieux.

J'ai aussi eu l'occasion de m'entretenir avec un étudiant japonais, SHU, en première année à l'université de Tokyo. Il est entré à l'université cet année, et il a choisi de participer au Programme FLY (Freshers' Leave Year Program, année sabbatique pour les étudiants de première année) (http://www.u-tokyo.ac.jp/stu01/PDF/flyprogram_2013.pdf) qui permet aux étudiants de première année de prendre une année sabbatique pour clarifier et confirmer leur motivation, et le but de leur études, en ayant des activités non universitaires; il peut 
Vivre en français en France Des japonais qui se cherchent

K. KomatsU

ENTRETIEN

AVEC SHU s'agir d'une expérience de stage, d'un séjour dans un pays étranger, etc. SHU est allé en Angleterre pour étudier l'anglais pendant un mois, avant de venir à Strasbourg pour sept mois pour étudier le français a l'IIEF (Institut International d'Études Françaises à l'Université de Strasbourg).

- Pourquoi êtes-vous venu en France? Dites-moi le but de votre venue en France.

SHU: Je suis venu en France pour étudier le français et je voulais vivre seul en Europe.

- Pourquoi étudiez-vous le français? Ou bien, voulez-vous étudier le français?

SHU: Je suis intéressé par la langue, et je voulais étudier une langue autre que l'anglais.

- Je vois... Donc pourquoi vouliez-vous vivre seul en Europe?

SHU: Si j'envisage de vivre à l'étranger dans le futur, je peux dire que c'est un essai, ou une expérience.

- Une expérience... S'il s'agit d'une expérience, elle est assez longue... Par vivre en Europe dans le futur, voulez-vous dire que vous aimeriez y vivre? Ou pensez-vous que c'est quelque chose qui pourrait vous arriver en général?

SHU: Je voulais vivre en Europe, et j'essaie simplement de le faire. Mes chances de vivre au Japon ou en Europe sont peut-être cinquante-cinquante.

- Si vos chances de vivre au Japon ou à l'étranger sont de cinquante-cinquante, est-ce lié à ce que vous voulez faire dans le futur, ou est-ce lié à votre travail, votre rêve, ou simplement comme ça?

SHU: Juste comme ça... Si je trouve un travail normalement, ce pourrait être dans une compagnie d'import-export, ou dans la fonction publique... dans ce cas, je serais intéressé par quelque chose lié à l'étranger ou à une organisation internationale.

- Donc c'est un essai parce que vous souhaitez vivre à l'étranger?

SHU: Plus ou moins.

- Pourquoi voulez-vous vivre en Europe dans le futur?

SHU: Je ne peux pas vraiment dire car il s'agit plus d'une pensée par élimination, si je peux dire, c'est juste un rêve, et même si je vis en Europe, je n'ai rien de particulier à faire en Europe, et cela dépend de comment la mondialisation évolue, et si nous avons plus de choix pour partir, cela pourrait être bien de vivre en Europe.

- Donc, vous vouliez vivre en Europe, pourriez-vous me dire plus précisément pourquoi vous êtes venu en France, et ce que vous 
Vivre en français en France Des japonais qui se cherchent

K. Komatsu
CONCLUSION voulez faire en France?

SHU: Je suis venu en France pour étudier le français. Les principales langues étrangères sont l'anglais, le français, l'espagnol, le chinois, et j'ai pensé pourquoi pas le français.

- Donc c'est parce que le français vous intéressait, et vous vouliez étudier une langue autre que l'anglais, non? Alors, comment se passe la vie en Europe?

SHU: Oui, c'est cela. Et je vis près de l'université au Japon donc il est difficile de vivre seul, donc si je veux (vivre seul), ce pouvait être en dehors du Japon. Et je voulais le faire tôt. Je peux plus ou moins me débrouiller pour vivre seul, cela n’a rien de spécial, et je m'en sors. Mais je ne sais pas trop comment cela se serait passé si j'avais dû trouver un logement moi-même (8).

- Donc que voudriez-vous faire avec la langue française dans le futur? Ou voulez-vous faire quelque chose en français?

SHU: Si je parle dans le futur, c'est difficile... Mais j'espère que cela pourrait être lié à mon travail. Pour l'instant, la langue française est juste un de mes intérêts, un de mes loisirs, et ce n'est pas quelque chose de primordial dans ma vie. Cela peut être utile mais rien de plus que cela.

- Que voudriez-vous faire après votre séjour en France? Quel est votre rêve ou objectif futur après votre séjour en France?

SHU: Ce n'est pas encore décidé. J'y réfléchirai lorsque je reviendrai au Japon. Comme je suis déjà venu ici, je pense qu’il est possible que je revienne ici.

Pour SA, la vie a été facile, c'est le concours d'entrée au Lycée qui lui avait demandé le plus d'efforts. Cependant elle sait que l'avenir lui demandera d'en fournir encore d'autres, et elle voulait se mettre dans une situation où elle devait faire des efforts pour réussir.

Une chose importante à remarquer est qu'elle est venue en France pour faire un effort ou pour se donner du courage comme l'indique le mot 'ganbaru' en japonais. En effet, on utilise ce verbe à l'impératif, «Ganbare», pour encourager par exemple les athlètes aux Jeux Olympiques.

Pour SHU, comme pour SA, il s'agissait d'un essai pour vivre seul dans un pays étranger, et comme il s'intéressait au français il a décidé de venir en France. Les deux étudiants apprennent la langue française comme un loisir: l'apprentissage du français est simplement une raison pour venir viore en France. Nous n'avons pas eu l'impression qu'ils apprennent le français pour leur futur ou leur travail. 
Vivre en français en France Des japonais qui se cherchent

K. Komatsu

\section{RÉFÉRENCES BIBLIOGRAPHIQUES:}

FURUKAWA C. 2005. The Possibility of Institutional Support for Fibun-Sagashi (Finding Oneself): A Case Study of the Gapyear in the U. K., Focusing on the Social Awareness Behind the Popularity of the Gapyear, The journal of educational sociology 77: 2746.

MIZUMA R. 2006. PCO jibun sagashi no soyo ni tuite (2) Charactaristics of jibun sagashi (looking for ourselves), Annual convention of the Japanese Association of Educational Psychology (48), 220.

NAKAMA R. 2008. Fibun Sagashi ruikeika no kokoromi to sorezore no tokucho ni tsuite- jikoiwakan to jikokaitakuishiki no wakugumi karano kento A study on types of self-exploration patterns and their features: From two axes regarding feelings of self-incoherence and intentions to self-development. University of Fukushima $\mathrm{N}^{\circ} 4$.

OHTA Y et ISHINO Y. 2011. Self-seeking for Undergraduates: An Investigation in relation to Self-Understanding and Grade Difference, Memoirs, Faculty of Education, Shimane University 45, 63-69.

ONO T. 2011. Identity no saikotei - Asia o tabi suru nihon jin backpacker no "jibun sagashi" no kiketu. Re-affirming identity: Fapanese backpackers in Asia and the consequence of their "self-seeking" travels, University of Kansai Gakuin, Department of Sociology (111), 155-170, Kansai Gakuin Daigaku Syakaigakubu Kenkyukai.

Embassy of the USA

http://japan2.usembassy.gov/amview/j/amview-j2010100182.html

Fukuoka women's University

http://www.fwu.ac.jp/international/program.php

Study abroad International Cross-cultural committee: http://www.daigaku-ryugaku.com/

Study abroad program

http://www.daigaku-ryugaku.com/08voice/01us/0201/v.html Univ. of Hirosaki

http://www.hirosakiu.ac.jp/kokusai/ni/kai/exp/chi/harupin_shi noda.pdf 


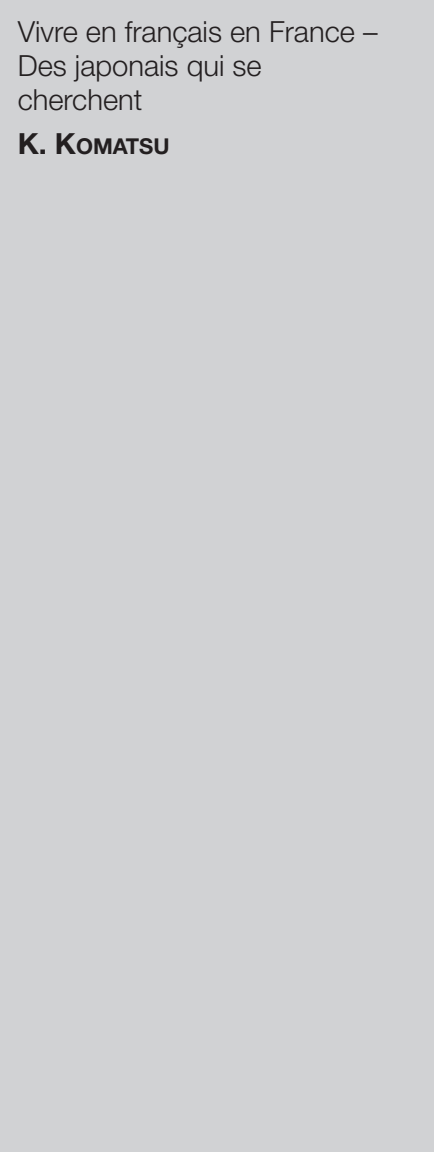

\section{NOTES}

* Pour créer un effet de décalage réflexif chez le lecteur, nous avons choisi de présenter la traduction des formulations significatives de la façon suivante: traduction mot à mot imagée et/ou énigmatique en italiques (mot ou expression du japonais en italiques: expression équivalente en français en caractères romans).

(1) http://business.nikkeibp.co.jp/article/skillup/20071126/141520/

(2) http://japan2.usembassy.gov/amview/j/amview-j20101001-82.html

(3) http://www.fwu.ac.jp/international/program.php

(4) http://www.daigaku-ryugaku.com/08voice/01us/0201/v.html

(5) Au cours de la visioconférence «Enseigner le Français Langue Etrangère dans le réseau culturel français en Chine et au Japon» qu'il a présentée le 11 décembre 2013, au Département de Didactique des Langues et du FLE-FLS-FLI de l'Université de Strasbourg.

(6) momareru est un verbe, à la voix passive, qui au sens propre, signifie «être bousculé», ou «être balloté» - au sens figuré, il s'agit d'être poussé hors de sa zone de confort par les événements)

(7) ganbaru est un verbe qui signifie «faire des efforts», «faire de son mieux». À l'impératif il peut se traduire en français par l'expression «Courage!».

(8) SHU a reçu de l'aide d'une amie résidant à Strasbourg pour trouver son logement. 